Document downloaded from:

http://hdl.handle.net/10251/140906

This paper must be cited as:

Salt Llobregat, JJ.; Alcaina-Acosta, JJ. (04-0). Dual-rate sampled-data systems. Some interesting consequences from its frequency response analysis. International Journal of General Systems. 48(5):554-574. https://doi.org/10.1080/03081079.2019.1608984

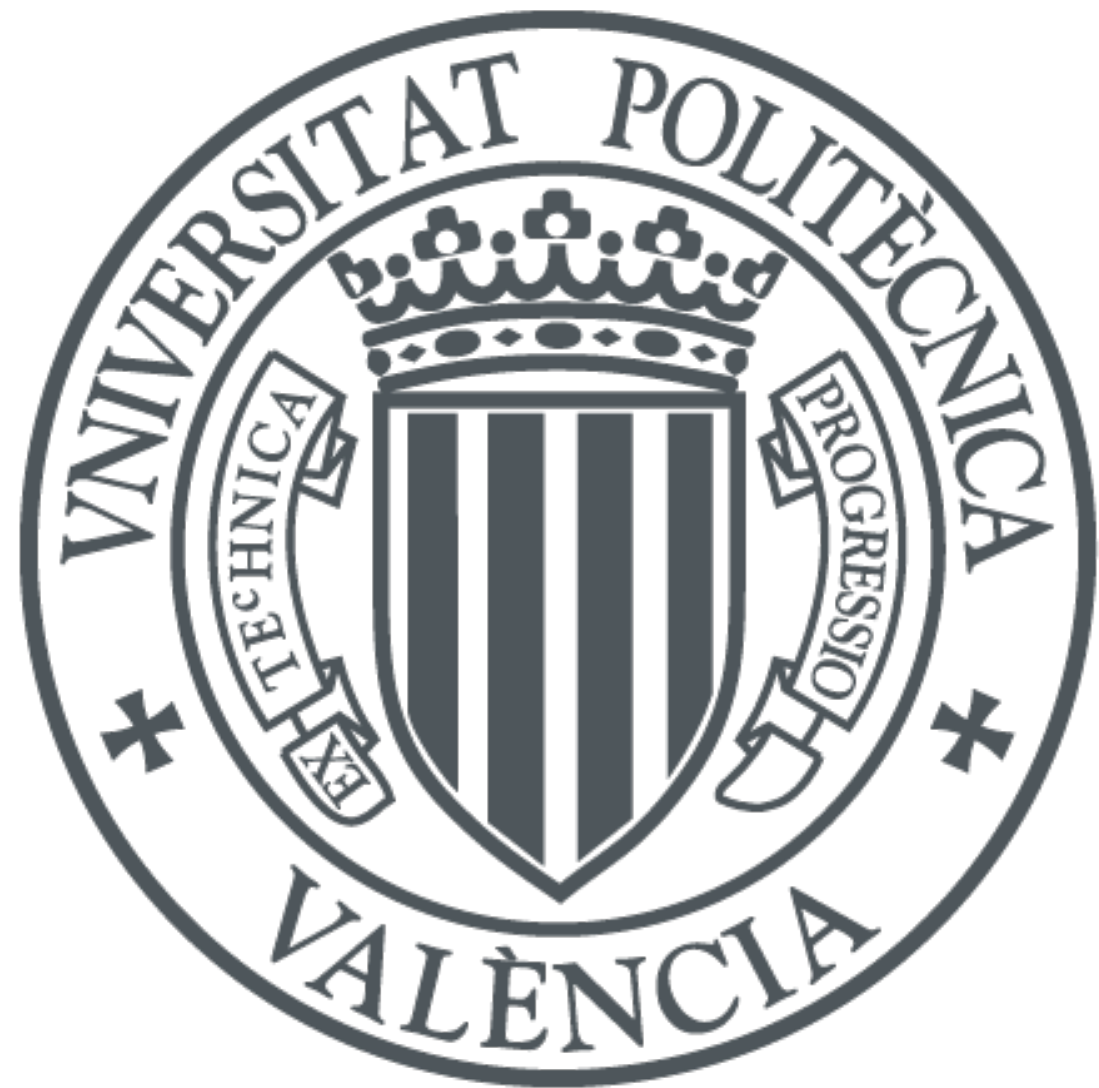

The final publication is available at

https://doi.org/10.1080/03081079.2019.1608984

Copyright Taylor \& Francis

Additional Information

This is an Accepted Manuscript of an article published by Taylor \& Francis in International Journal of General Systems on JUL 4 2019, available online: http://www.tandfonline.com/10.1080/03081079.2019.1608984 


\title{
ARTICLE
}

\section{Dual-Rate Sampled-Data Systems. Some interesting consequences from its Frequency Response Analysis}

\author{
Julián Salt, José Alcaina \\ Instituto de Automática e Informática Industrial. Universitat Politècnica de València. Spain
}

\begin{abstract}
ARTICLE HISTORY
Compiled March 12, 2019

ABSTRACT

The main goal of this contribution is to introduce a new procedure in order to analyze properly SISO dual-rate systems (DRS) and to provide straightforward answers to some common general questions about this kind of systems. Frequency response analysis based on DRS lifting modeling can lead to interesting results about stability margins or performance prediction. As a novelty, it is explained how to understand DRS frequency response and how to handle it for an easy computation of magnitude and phase margins keeping classical frequency domain methods. There are also some repetitive questions about DRS that can be analyzed and answered properly using the results from this contribution: what the optimum relation between sampling periods is or what effects does delay have in a DRS . Every step is illustrated with examples that should clarify the understanding of the text.
\end{abstract}

\section{KEYWORDS}

Dual-rate systems, frequency response, stability, Bode diagram

\section{Motivation}

In general a multi-rate sampled data system (MRS) is defined as a hybrid system composed of continuous time elements, usually the plant, and some discrete time components, usually the controllers and/or the filters, where two or more variables are sampled or updated at different frequencies. These systems have been reported and applied in fields like signal processing and communications for a long time. In digital control systems MRS were assumed by engineers since the fifties with seminal papers like (Kranc 1957; Friedland 1959; Coffey and Williams 1966; Kalman and Bertram 1959). When there are just two sampling periods, the system is called dual-rate. The consideration of this kind of systems is proposed mainly due to restrictions in sampling periods. For instance slow chemical measurements or artificial vision sensing where the processing data is time consuming. This situation leads to unacceptable sampling times for control purposes in a single rate enviroment. In networked control systems (NCS) it is a viable solution either to avoid delays (Cuenca et al. 2011) or to save network load by sending less information across the shared medium. Due to the current importance of the NCS applications, other non-conventional sampling schemes (like DRS) for dealing with communication bandwidth restrictions, such as event-triggered sampling, have been lately proposed (Hou et al. 2018; Bu et al. 2018; Zhao et al. 2018; Liu, 
Liu, and Alsaadi 2016). In some low cost devices, DRS is also necessary for solving problems due to the low resolution of sensors (cheap encoders) (Petrella and Tursini 2008). These DRS cases are what is defined as MRIC (multi-rate input control) that is a slow output but a higher rate input which is the most common schema in DRS. It is usual that both sampling periods are related by a natural number $N \in \mathbb{N}$. A regular pattern of sampled signals without sampling times mismatch between them will also be considered.

In the field of control systems, there are two trends for modelling MRS (and DRS). It can be said that one of them is an external representation based on (Tsai, Chen, and Shieh 1993; Cimino and Pagilla 2010b,a; Du et al. 2011) and the other one is a state space form. These options consider the least common multiple of all sampling periods for modelling purposes ${ }^{1}$. In the external case the skip (downsampling) and expand (upsampling) operations are crucial and in the state space form a MIMO model is assumed enveloping the input and output of every system in vectors according to the frame period (least common multiple). The system progresses repeating the same scheme frame after frame. Therefore this MR (time-varying system) can be transformed into a LTI system once the system description is enlarged over a "metaperiod". Using this technique, even from a single-input single-output (SISO) system an artificial multi-input and multi-output (MIMO) system is obtained. This procedure is known as lifting in control area (Bamieh et al. 1991; Chen June 2005; Albertos 1990), originally denoted as Vectorial Switch Decomposition by (Kranc 1957) or blocking (in signal processing) (Meyer and Burrus 1975).

Consider now a SISO system with two samplers (DRS); in these conditions lifting provides a MIMO system. A classical issue in multi-rate control schemes is that such a MIMO lifted system can be managed as any other multivariable MIMO one. However, there are not different input and output variables, but just one input and one output, "lifted" at different input and output sampling times in a periodically-repeating metaperiod. This will be a very important aspect when a DRS frequency response is considered. Usually classical MIMO techniques are considered -it is common to study the frequency response of dual-rate plants by using singular value decomposition (SVD) of the lifted MIMO system-, losing important features that will be introduced in this contribution. In order to solve this problem a new algorithm for DRS frequency response computation in discrete control systems was introduced by (Salt and Sala 2014), but it seems difficult to understand the complete meaning from that reference, which will be clarified now. It has been studied how this technique can be advantageous by obtaining the frequency response and its derived concepts and procedures when applying to DRS. In fact, this is the main goal of this article and some examples are introduced to emphasize its importance. Specifically it will attempt to meet answers to repetitive questions made when a DRS is analyzed: what is the best relation between sampling periods or about the influence of delay in these systems.

The structure of the paper is as follows: the next section introduces some preliminary material and exposes how to compute the frequency response for a DRS in an efficient way; Section 2 presents how to make this frequency response easily understandable introducing an example. In the following section, the results from the previous section are used to explain the advantages of DRS frequency response and how to answer some classical questions about the optimum value of $N$ in a simple MRIC DRS. A conclusion section closes the paper.

\footnotetext{
${ }^{1} \mathrm{~A}$ more formal definition will be given in section 2
} 


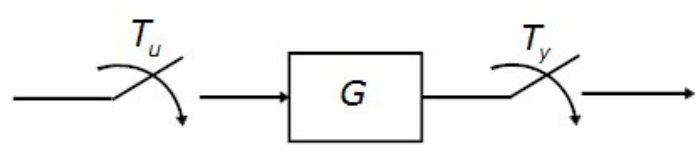

Figure 1. Generalized Dual-Rate System

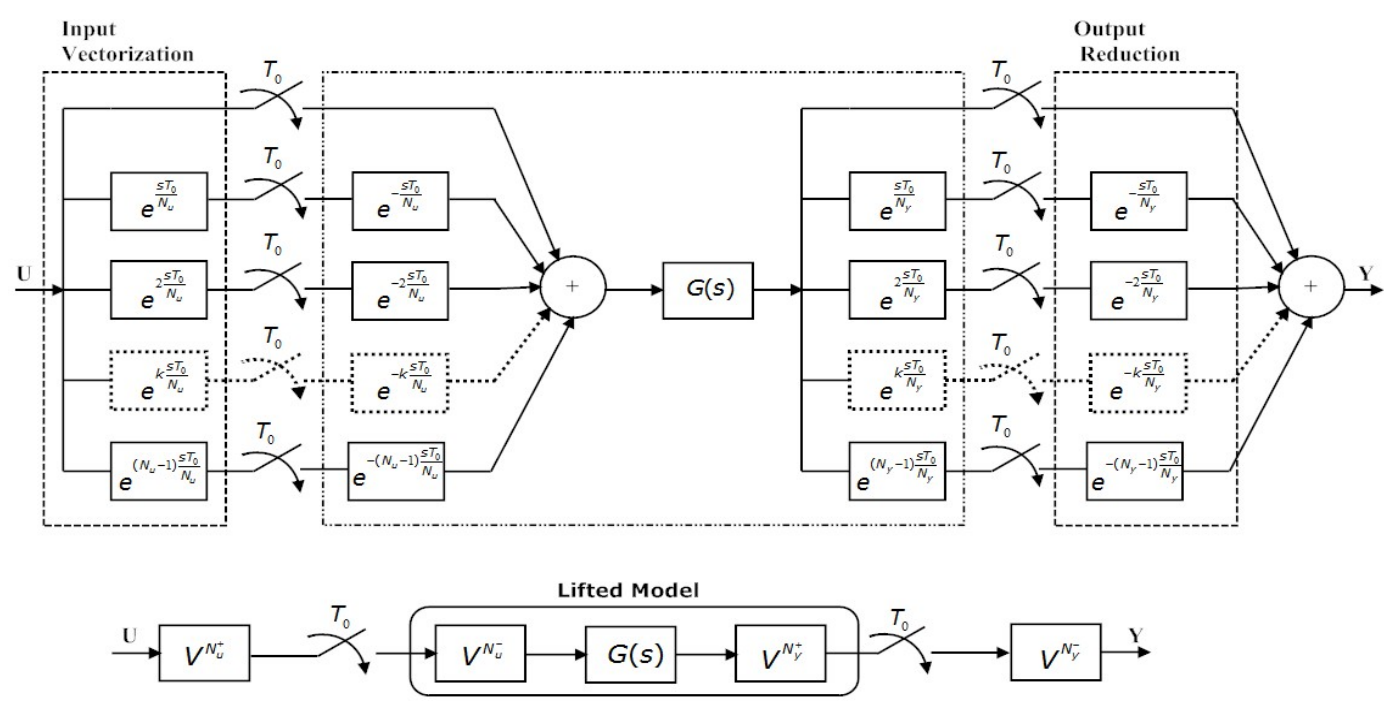

Figure 2. Kranc's vector switch decomposition

\section{DRS frequency response computation: a classical interpretation}

\subsection{Preliminaries and notation}

A dual-rate discrete LTI system is one in which the input and output sequences are assumed to have different sampling periods, $T_{u}$ and $T_{y}$. If they are rationally related, it is possible to define $T_{0}$ as the minimum real number such that $T_{0} / T_{u}$ and $T_{0} / T_{y}$ are integers. It is similar to a least common multiple $T_{0}=\operatorname{lcm}\left(T_{u}, T_{y}\right)$ whose definition related to real numbers can be confusing. $T_{0}$ is usually known as "metaperiod" or "frame period" and integers $N_{u}, N_{y}$ can be defined complying with $T_{0}=T_{u} N_{u}=T_{y} N_{y}$ (indeed, then $T_{u} / T_{y}=N_{y} / N_{u}$ is a rational number). It is also usual to define $T$ as a kind of "greatest common divisor sampling period", such that $T_{0}=N T$ being $N=\operatorname{lcm}\left(N_{u}, N_{y}\right)$; therefore $T_{0}=N T=N_{u} T_{u}=N_{y} T_{y}$. With these conditions, the behaviour of the DRS may be characterised via a "lifted" transfer function matrix

$$
y_{l}\left(z^{N}\right)=G_{l i f t e d}\left(z^{N}\right) u_{l}\left(z^{N}\right)
$$

where the variable $z$ stands for the LTI $z$-transform argument at sampling period $T$, and consequently $z^{N}$ is related to $T_{0}=N T$. In equation (1) $y_{l}$ is a vector of length $N_{y}$, $u_{l}$ is a vector of length $N_{u}$ and $G_{l i f t e d}$ is a $N_{y} \times N_{u}$ transfer function matrix (Bamieh et al. 1991). The lengths of the vectors are increased in the case of MIMO systems (multiplied by the number of outputs and inputs, respectively). The next figures can help to completely deduce and clarify this equation.

Figure 1 depicts an open loop DRS and figure 2 describes the application of Kranc's idea vector switch decomposition to the situation of figure 1 . Note that in figure 2, $T_{0} / N_{u}=N_{y} T$ and $T_{0} / N_{y}=N_{u} T$. It can be seen that with this scheme, the central 
part of the diagram would be

$$
\left(1 z^{-N_{u}} \ldots z^{-\left(N_{y}-1\right) N_{u}}\right) \frac{G_{l i f t e d}\left(z^{N_{y} N_{u}}\right)}{N_{y}}\left(\begin{array}{c}
1 \\
z^{N_{y}} \\
\vdots \\
z^{\left(N_{u}-1\right) N_{y}}
\end{array}\right)
$$

This expression can be also derived using the known Mason's rule for blocks diagram. This diagram is now a $T_{0}$ single rate blocks diagram ${ }^{2}$. Actually, that is the main reason for Kranc's procedure.

\subsection{Sampled-data lifted systems}

There are different ways to obtain the lifted model. For processes, it may be that the easiest one is to consider the successive iterations from the gcd $T$ discrete state space. If a strictly proper continuous system is discretised (assuming $\mathrm{ZOH}$ ) at period $T$, with a realization $(A, B, C, 0)$, then the lifted dual-rate model has a realization $\left(A_{l}, B_{l}, C_{l}, D_{l}\right)$, at metaperiod $T_{0}$, where these matrices are obtained by repeated evaluations of the equations at sampling period $T$ giving rise to well-known convolution-like formulae. For instance

$$
\begin{aligned}
y\left(k T_{0}+\zeta T\right)= & C x\left(k T_{0}+\zeta T\right)= \\
& =C\left[A^{\zeta} x\left(k T_{0}\right)+A^{\zeta-1} B u\left(k T_{0}\right)+\right. \\
& \left.+A^{\zeta-2} B u\left(k T_{0}+T\right)+\ldots+B u\left(k T_{0}+(\zeta-1) T\right)\right]
\end{aligned}
$$

for $\zeta=1, \ldots,\left(N_{u}-1\right) N_{y}$, However, the zero-order-hold entails

$$
\begin{aligned}
& u\left(k T_{0}+d N_{y} T\right)=u\left(k T_{0}+\left(d N_{y}+1\right) T\right)=\cdots=u\left[k T_{0}+\left((d+1) N_{y}-1\right) T\right] \\
& \forall d=0,1 \ldots,\left(N_{u}-1\right)
\end{aligned}
$$

Therefore the lifted matrices are obtained by suitably stacking the results from the above equation. It is also possible to consider that $\mathrm{G}$ has different natures (continuous system without $\mathrm{ZOH}$, digital system, etc). For each of these cases a proper input or output assignment (like (4)) must be established leading to a specific lifted model (Thompson 1986). Some other details, omitted here for brevity, can be found in (Bamieh et al. 1991; Albertos 1990; Salt, Torneno, and Albertos 1993). Now, a brief example could be considered. If in figure $1 G$ is a continuous-time plant with $\mathrm{ZOH}$ and $T_{u}=0.3$ y $T_{y}=0.2\left(N_{u}=2\right.$ and $\left.N_{y}=3\right)$, then the $\mathrm{lcm} T_{0}=0.6$, the gcd $T=0.1$, and the lifted representation will be

${ }^{2}$ It will denoted $w_{s}=2 \pi / T_{0}$ 


$$
\begin{aligned}
& x\left[(k+1) T_{0}\right] \quad=A^{6} x\left(k T_{0}\right)+\left(\begin{array}{ll}
\left(A^{5}+A^{4}+A^{3}\right) B & \left.\left(A^{2}+A+I\right) B\right)
\end{array}\right)\left(\begin{array}{c}
u\left(k T_{0}\right) \\
u\left(k T_{0}+3 T\right)
\end{array}\right) \\
& \left(\begin{array}{c}
y\left(k T_{0}\right) \\
y\left(k T_{0}+2 T\right) \\
y\left(k T_{0}+4 T\right)
\end{array}\right)=\left(\begin{array}{c}
C \\
C A^{2} \\
C A^{4}
\end{array}\right) x\left(k T_{0}\right)+\left(\begin{array}{cc}
0 & 0 \\
C(A+I) B & 0 \\
C\left(A^{3}+A^{2}+A\right) B & C B
\end{array}\right)\left(\begin{array}{c}
u\left(k T_{0}\right) \\
u\left(k T_{0}+3 T\right)
\end{array}\right)
\end{aligned}
$$

\subsection{DRS Frequency Response}

From a lifted LTI representation of a DRS, it is possible to calculate the frequency response. The Theorem introduced in (Salt and Sala 2014) says: The output $y(k)$, when $u(k)=e^{j \omega T_{u} k}$, of a SISO dual-rate $\left(N_{u} T_{u}=N_{y} T_{y}\right)$ lifted system $y_{l}(z)=G_{\text {lifted }}(z) u_{l}(z)$ is a collection of components $y_{r}(k)=\bar{y}_{r} e^{j T_{y} \omega_{r} k}$ of frequencies $\omega_{r}=\omega+2 \omega_{y}^{s} r / N_{y}$, for $r=0, \ldots,\left(N_{y}-1\right)$, with $\omega_{y}^{s}=\pi / T_{y}$, and $\bar{y}_{r}$ is given by

$$
\bar{y}_{r}=\frac{1}{N_{y}} \sum_{p=0}^{N_{y}-1} \sum_{q=0}^{N_{u}-1} G_{p q}\left(e^{j \omega_{r} T_{y} N_{y}}\right) e^{-j T_{y} \omega_{r} p} e^{j \omega T_{u} q}
$$

The singe-rate discrete frequency-response computations are carried out by replacing $z=e^{j \omega T}$ for some $T$. It is easy to check that in the DRS case, from (6), the components will be given by the product of the frequency response of the left factor

$$
\left[1 z^{-1} z^{-2} \ldots z^{-\left(N_{y}-1\right)}\right] G_{l i f t e d}\left(z^{N_{y}}\right)
$$

replacing $z=e^{j \omega_{r} T_{y}}$, which gives a row vector, and the right factor (column vector)

$$
\left(1 z z^{2} \ldots z^{N_{u}-1}\right)^{T}
$$

replacing $z=e^{j \omega T_{u}}$.

As it will be explained later, there is a possibility of computing the whole frequency response from only one Bode plot in the case in which $N_{y}$ and $N_{u}$ are coprime. Note that, $\omega_{s}=2 \pi /\left(N_{y} T_{y}\right)$ and then that the components of the frequency response are defined at $\omega_{r}=\omega+r \omega_{s}$, for, $r=0, \ldots, N_{y}-1$. For more details, see (Salt and Sala 2014).

\section{Frequency Response Interpretation}

As it is relatively easy to understand considering the equation (2) and unwrapping it, that is, doing the matrices products, an usually high dimension SISO transfer function is reached. This is a simple procedure but it produces very unsatisfactory results as will be shown later in this section. The results from the algorithm proposed in the previous section are proper but require an interpretation. From the results above, it can be tested that the DRS frequency response can be explained by adding $\frac{N_{y}}{\operatorname{gcd}\left(N_{u}, N_{y}\right)}$ values from the Bode diagram outlined from 0 to $\left(\frac{N_{y}}{\operatorname{gcd}\left(N_{u}, N_{y}\right)}-1\right) N_{u} w_{s}$. In fact, for an input frequency $b \mathrm{Rad} / \mathrm{s}$, the output is the sum of sine signals with frequencies $b$, 

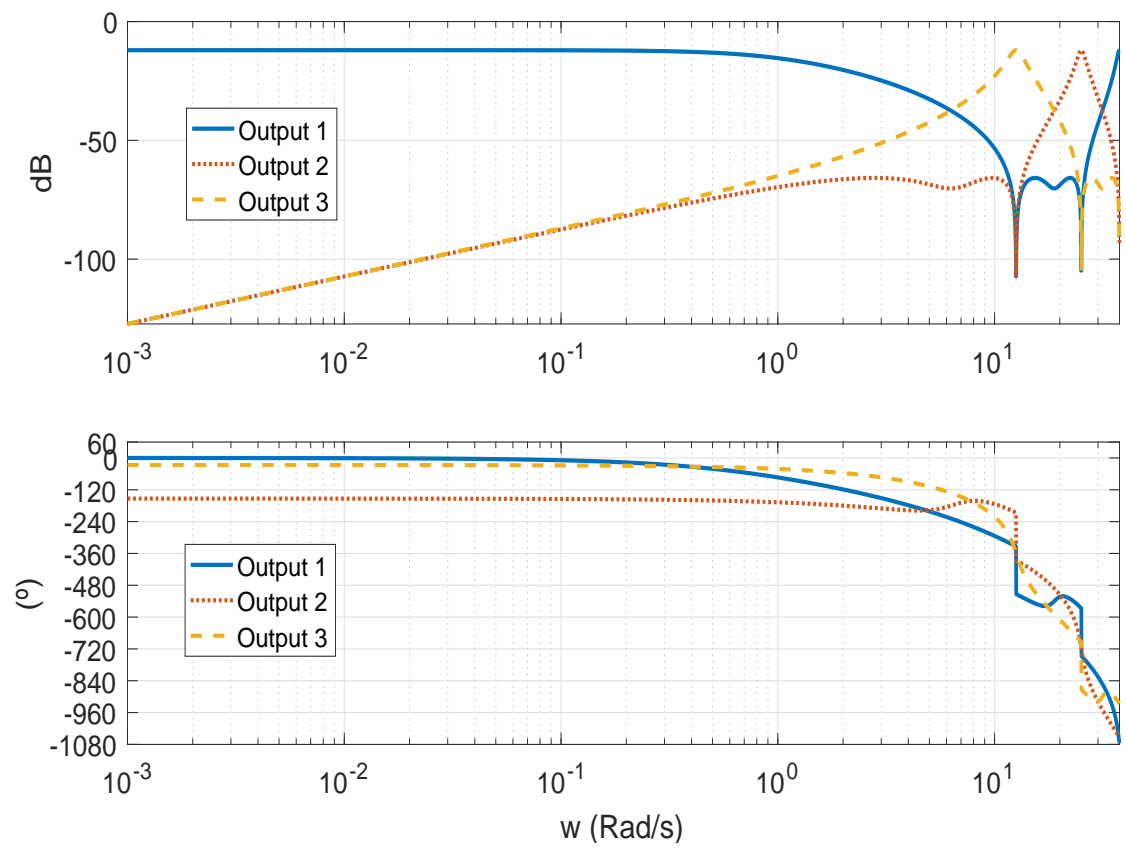

Figure 3. DRS Bode

$b+N_{u} w_{s}, b+2 N_{u} w_{s}, \ldots b+\left(\frac{N_{y}}{g c d\left(N_{u}, N_{y}\right)}-1\right) N_{u} w_{s}$ with amplitude and phase determined by Bode diagram at the indicated points. The coprime case is illustrative and gives a link with the theoretical results. If $N_{u}$ and $N_{y}$ are coprime, then, considering the blocks diagram from figure 2, the DRS frequency response is obtained reading the $N_{y}$ points from the magnitude and phase Bode diagrams in the interval from 0 to $N_{u} N_{y} w_{s}$, each length $N_{u} w_{s}$.

The proposed algorithm introduced in (Salt and Sala 2014) returns $N_{y}$ Bode diagrams concerning to the tracks: 0 to $N_{u} N_{y} w_{s}, N_{u} w_{s}$ to $\left(N_{y} N_{u}+N_{u}\right) w_{s}, \ldots$, $\left(N_{y}-1\right) N_{u} w_{s}$ to $\left(N_{y} N_{u}+\left(N_{y}-1\right) N_{u}\right) w_{s}$. So, the same Bode translated $N_{u} w_{s} R a d / s$ $N_{y}$ times is reached. Of course, the $N_{y}$ points can be read in the interval 0 to $N_{u} w_{s}$ knowing the true sense of all of them.

In the following section, a simple example is introduced illustrating the procedure. The academic system $G(s)=\frac{1}{(s+1)(s+4)}$ preceded by a zero order hold is considered, with $N_{u}=2$ and $N_{y}=3$ and a metaperiod $T_{0}=1$, that is $T_{u}=1 / 2$ and $T_{y}=1 / 3$. The DRS frequency response is formed by three components. The result is showed in figure 3. For this case, $w_{s}=\frac{2 \pi}{T_{0}}=6.28 R / s, N_{u} w_{s}=12.566 R / s$ and $N_{u} N_{y} w_{s}=37.699 R / s$. As it can be seen, the Bode diagram is made up of three components whose responses are achieved each $N_{u} w_{s}$. In fact and as it was said before, if the input is a sine signal with pulsation $w=3 R / s$, the three components are:

\begin{tabular}{|c|c|c|c|}
\hline Component & $\mathrm{w}(\mathrm{R} / \mathrm{s})$ & Magnitude $(\mathrm{dB})$ & Phase $(\mathrm{Rad})$ \\
\hline 1 & 3 & -24.8 & -2.64 \\
\hline 2 & $3+12.56=15.56$ & -65.85 & 2.96 \\
\hline 3 & $3+\left(2^{*} 12.56\right)=28.3$ & -51.11 & -1.275 \\
\hline
\end{tabular}

In figures 4 and 5 the procedure is explained graphically. If the multiplicities $N_{u}, N_{y}$ are coprime it is possible to read every value in just one Bode diagram. This is the 

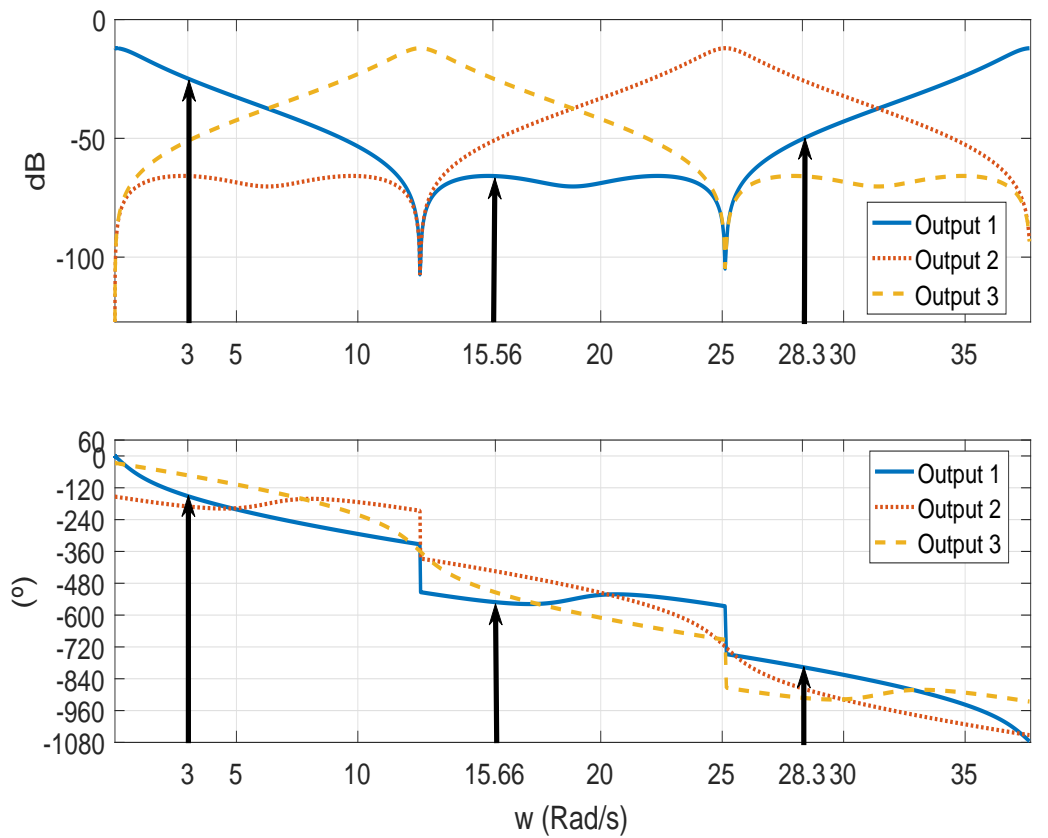

Figure 4. DRS Bode Interpretation. $w=3 R / s$

same as reading the value of each component in its proper $w$ and it is also the same as reading the 0 to $w_{s}$ zone but taking into account that the real value of the frequency of component $i$ is $w+i \times N_{u} w_{s}$. Note the linear scale of the frequency axis in both figures.

At this point, it is time to check the accuracy of the procedure. Adding the three responses, the result is equivalent to the output of the $Z O H-G(s)$ with the proposed input. As it can be seen in figure 6 the output true response is mapped at $T_{y}$ at steady state.

However, in the classical way, as it was said before, the problem is the high dimension of the transfer function that usually leads to an inefficient use of classical Bode computation routines. Some examples are now introduced for the explanation of this problem. If the continuous-time system with $\mathrm{ZOH}$

$$
G(s)=\frac{20}{(s+1)(s+3)}
$$

is considered, the results for a metaperiod $T_{0}=0.1 T_{u}=T_{0} / 2$ and $T_{y}=T_{0} / 3$ computed using both methods are plotted in figure 7 . As it is observed, the results from the procedure based on obtaining the transfer function show some differences with the correct ones.

The mistake is even greater if the degree increases, considering for instance a metaperiod $T_{0}=0.1, T_{u}=T_{0} / 4$ and $T_{y}=T_{0} / 3$. Figure 8 plots this last case with unacceptable results.

However a problem is found if the purpose is to work with these diagrams in a classical way obtaining important values such as margins for stability. The reason is that this sum is not really a pure sine signal. It is possible to assume a shortcut, 

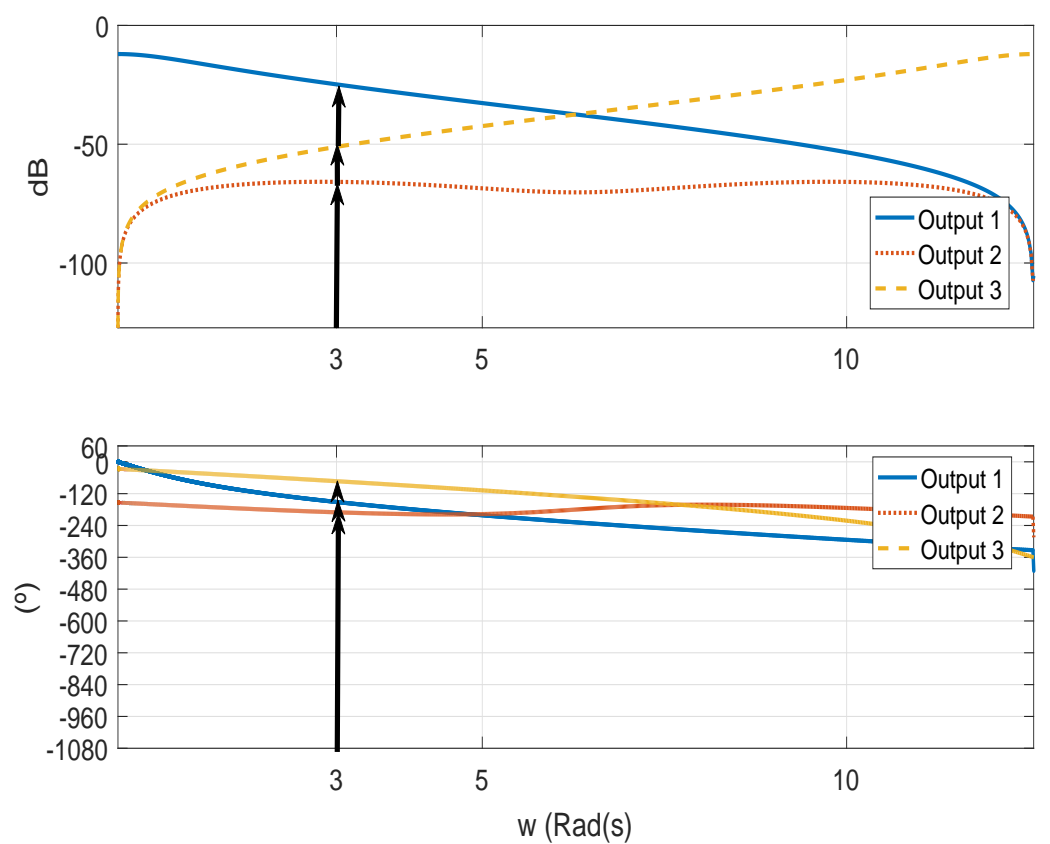

Figure 5. DRS Bode. $w=3 R / s$. Zoom

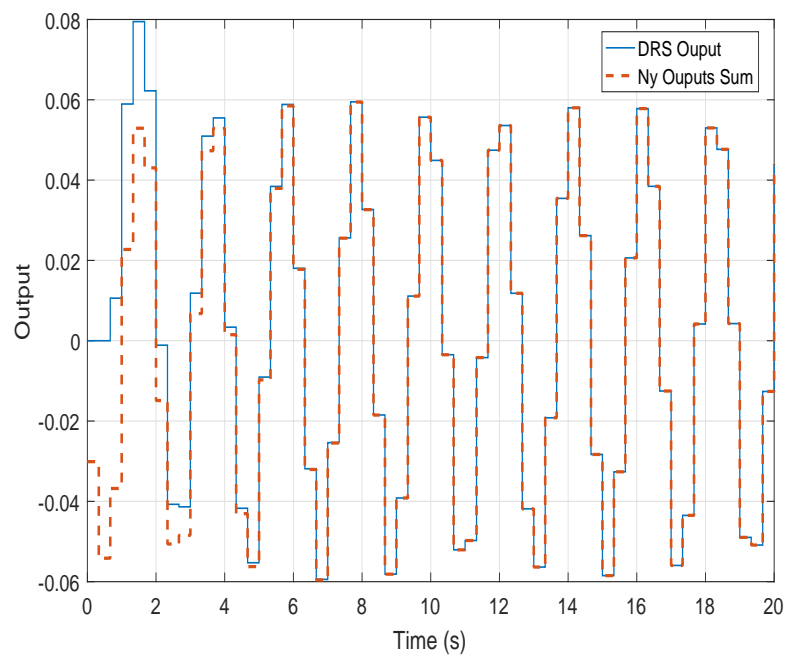

Figure 6. Output Components Sum Comparison 

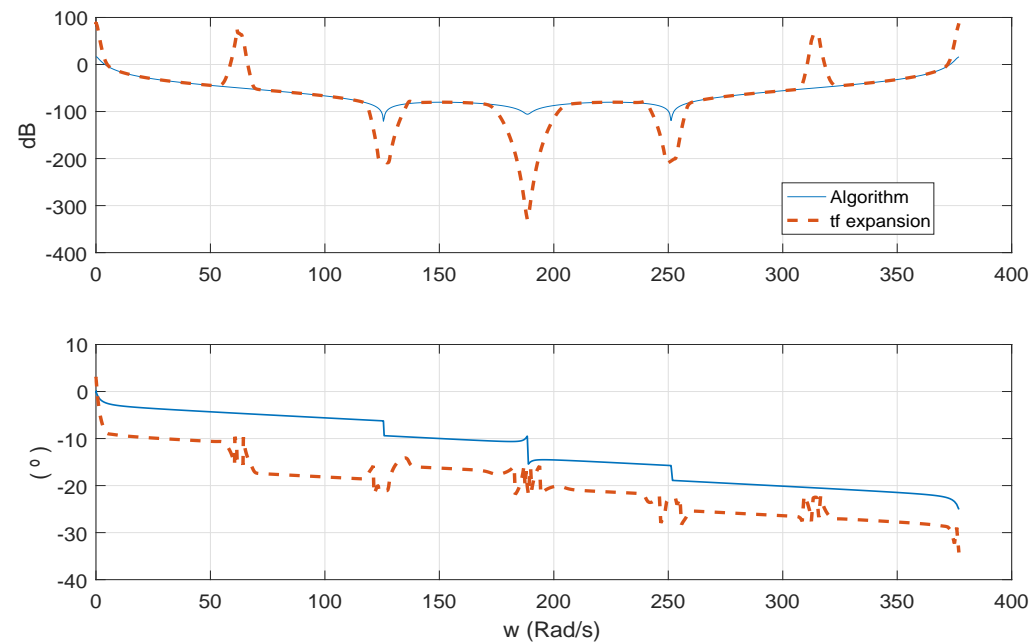

Figure 7. DRS Bode. Comparison of Methods
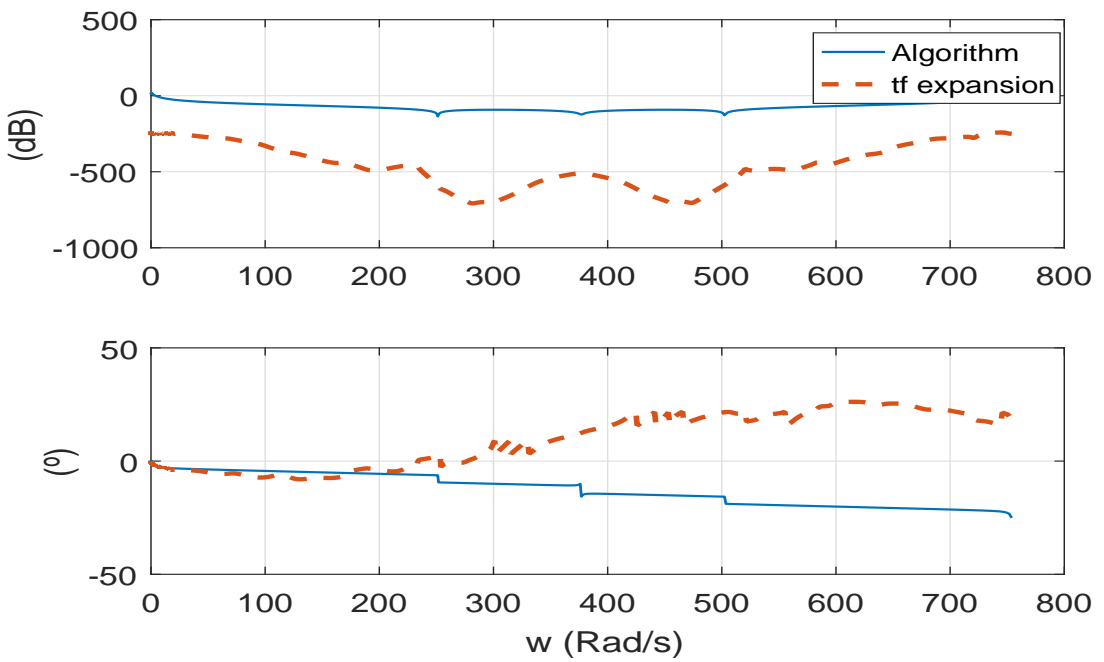

Figure 8. DRS Bode. Comparison of Methods 
adding the different output components at a specific metaperiod. In the case that the multiplicities $N_{u}, N_{y}$ were coprime, the output is ${ }^{3}$

$$
\begin{aligned}
y\left(k T_{y}\right)= & A_{1} \sin \left(w k T_{y}+\varphi_{1}\right)+A_{2} \sin \left(\left(w+N_{u} w_{s}\right) k T_{y}+\varphi_{2}\right)+\ldots+ \\
& +A_{N_{y}} \sin \left(\left(w+\left(N_{y}-1\right) N_{u} w_{s}\right) k T_{y}+\varphi_{N_{y}}\right)
\end{aligned}
$$

As it was said this is not a pure sine signal. However if the decomposition of each component is observed

$$
\begin{aligned}
A_{\nu} \sin \left(w_{\nu} k T_{y}+\varphi_{\nu}\right)= & A_{\nu} \sin \left(w_{\nu} k T_{0}+\varphi_{\nu}\right)+ \\
& z_{T_{y}}^{-1} A_{\nu} \sin \left(w_{\nu}\left(k T_{0}+T_{y}\right)+\varphi_{\nu}\right)+\ldots \\
& z_{T_{y}}^{-\left(N_{y}-1\right)} A_{\nu} \sin \left(w_{\nu}\left(k T_{0}+\left(N_{y}-1\right) T_{y}\right)+\varphi_{\nu}\right)+ \\
& \text { for } \quad \nu=1, \ldots,\left(N_{y}-1\right) \\
A_{\nu} \sin \left(w_{\nu} k T_{y}+\varphi_{\nu}\right)= & A_{\nu} \sin \left(w_{\nu} k T_{0}+\varphi_{\nu}\right)+ \\
& z_{T_{y}}^{-1} A_{\nu} \sin \left(w_{\nu} k T_{0}+\left(w_{\nu} T_{y}+\varphi_{\nu}\right)\right)+\ldots \\
& z_{T_{y}}^{-\left(N_{y}-1\right)} A_{\nu} \sin \left(w_{\nu} k T_{0}+\left(w_{\nu}\left(N_{y}-1\right) T_{y}+\varphi_{\nu}\right)\right)+ \\
& \text { for } \nu=1, \ldots,\left(N_{y}-1\right)
\end{aligned}
$$

So, adding the contributions of each component at $k T_{0}$, that is the skip of $y\left(k T_{y}\right)$

$$
\begin{aligned}
y^{T_{0}}\left(k T_{y}\right) & =A_{1} \sin \left(w k T_{0}+\varphi_{1}\right)+ \\
& \left.+A_{2} \sin \left(w+w_{s}\right) k T_{0}+\left(\left(w_{+} w_{s}\right) T_{y}+\varphi_{\nu}\right)\right)+\ldots \\
& +A_{N_{y}} \sin \left(\left(\left(w+\left(N_{y}-1\right) w_{s}\right)+\left(N_{y}-1\right) N_{u} w_{s}\right) k T_{0}+\varphi_{N_{y}}\right)
\end{aligned}
$$

This finally leads to the $T_{0}$ approximation

$$
\begin{aligned}
y^{T_{0}}\left(k T_{y}\right) & =A_{1} \sin \left(w k T_{0}+\varphi_{1}\right)+ \\
& +A_{2} \sin \left(w k T_{0}+\left(w_{s} T_{0}+\varphi_{\nu}\right)\right)+\ldots \\
& +A_{N_{y}} \sin \left(\left(w k T_{0}+\left(\left(N_{y}-1\right) w_{s} T_{0}+\varphi_{N_{y}}\right)\right)\right.
\end{aligned}
$$

The results of such approximation are depicted in figure 9 .

These time responses have been achieved by using the diagram in figure 10

\section{Stability Margins for DRS}

Once the DRS frequency response is known and its interpretation has been explained, the important concept of Stability Margins must be presented. In general, it could be said that the gain margin is the range of a gain $\alpha \in \mathbb{R}$ that multiplying the open

${ }^{3}$ Remember that in general there will be $\frac{N_{y}}{g c d\left(N_{u}, N_{y}\right)}$ 


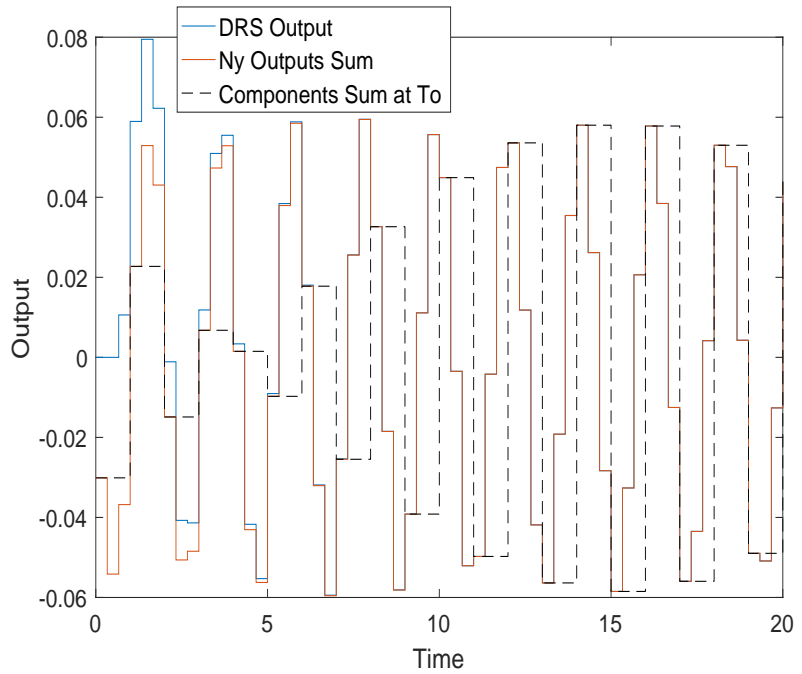

Figure 9. Output Components Sum at $T_{0}$ Comparison

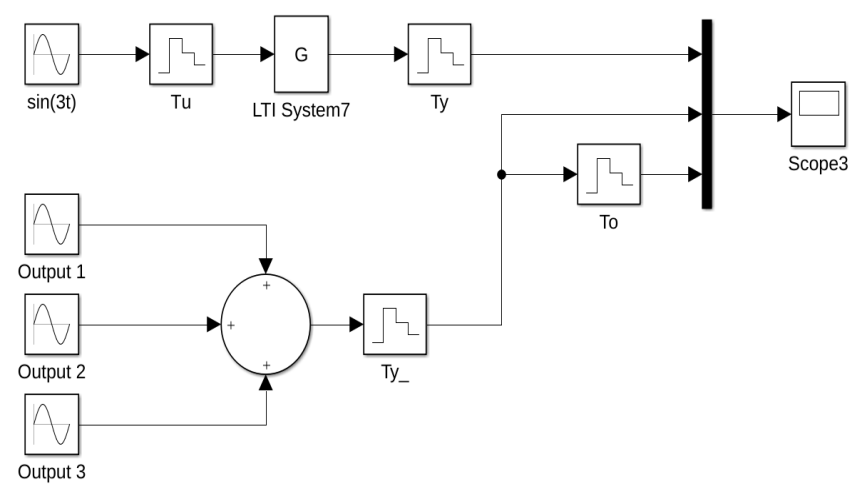

Figure 10. Simulation Diagram. Case $N_{y}=3$ 

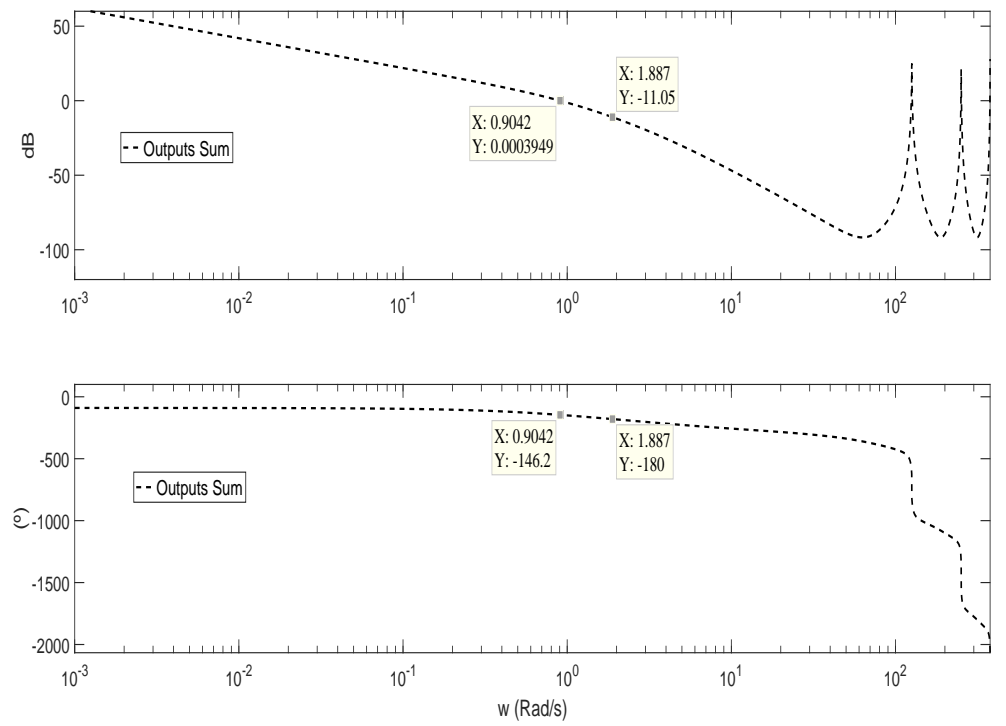

Figure 11. $T_{0}$ Bode Stability Margins Determination

loop transfer function system preserves the stability. In a Bode diagram it is usual to find the frequency for $a-\pi R a d$ and read the gain magnitude for that frequency. The interval from that value to $0 d B$ is the gain margin, obviously if the value is under $0 d B$. Regarding the phase margin, now the frequency for which the $0 d B$ is read and the interval from the angle for that frequency to $-\pi R a d$ is this phase margin. In this case there is an interval as well but the minimum value is the minus positive angle and for this reason just the maximum value is given (Thompson 1986) The same explanation could be applied for a DRS because it is possible to obtain a Bode diagram. As it was said before, this is just viable with the sum of all the output components for $T_{0}$ period (a metaperiod Bode). As it was explained it is an approximation, a very applicable one, as the practical experiences will show.

\subsection{Example}

Let us study the stability margins with the system

$$
G(s)=\frac{5}{s(s+1)(s+4)}
$$

with $T_{u}=T_{0} / 2$ and $T_{y}=T_{0} / 3$. The system is preceded by a ZOH. In this case, the $T_{0}=0.1$ metaperiod Bode diagram is depicted in figure 11 .

As it can be seen in figure 11 a gain margin of $11.05 \mathrm{~dB}$ and a phase margin of $33.8^{\circ}$ are obtained.

The top signal in figure 12 represents the simulation with a gain of $10\left(\frac{11.05}{20}\right)$ and the bottom signal shows the time response when the system has a critical time delay of 

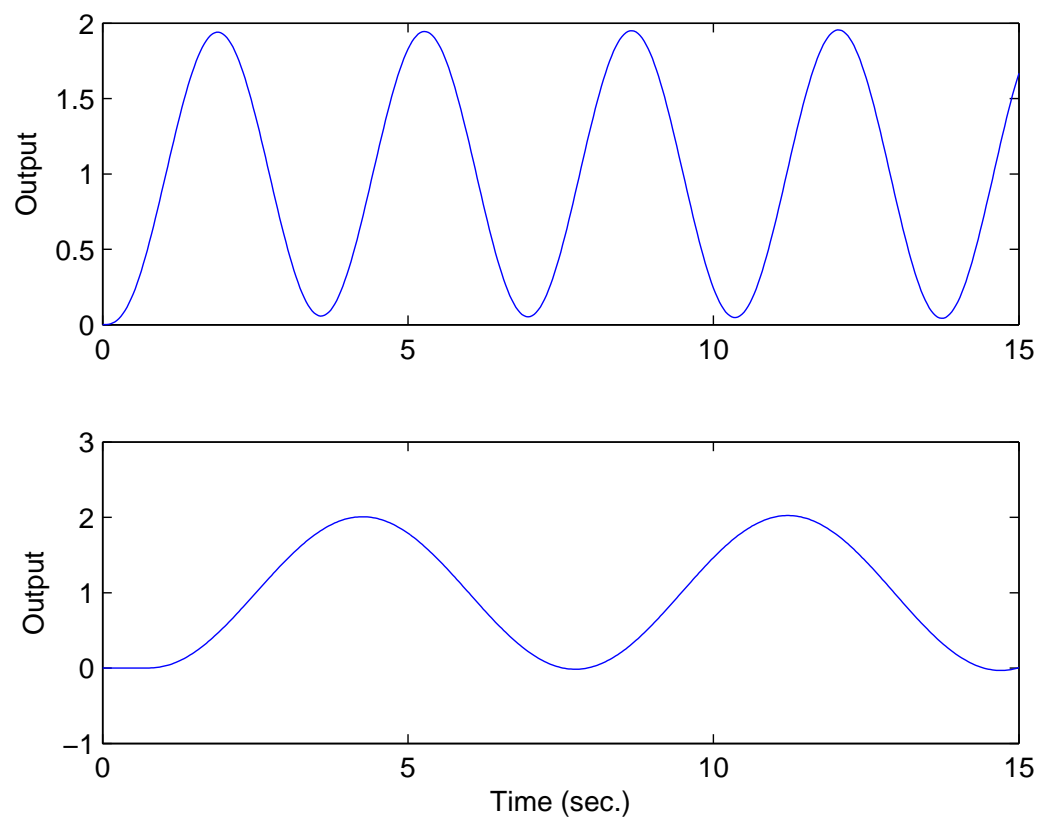

Figure 12. Simulation of DRS with Stability Margins consideration

$$
t_{\text {delay }}=\frac{(\text { Phase Margin })^{\circ}}{57.3 w_{0 d B}}=0.65 \mathrm{sec}
$$

Note that if the DRS is considered as a MIMO system and, as usual, the singular values of the MIMO transfer function frequency response are studied in this domain, it is more difficult to know the phase margin except when using a characteristic gain loci (Thompson 1986)

Figure 13 shows both the magnitude and the greatest singular value of the DRS frequency response in the metaperiod Bode diagram.

\section{Optimum $N$ Determination}

A question or a repetitive comment that every reader considers when she/he is reading a MRS contribution is: what is the best relation in a DRS when $T_{u}=T_{0} / N$ and $T_{y}=T_{0}$ ?, (that is in a MRIC scheme). Sometimes the $N$ is imposed by the specific problem but if the designer can choose the $N$ value, which will be the best one? Generally, at first, there is no way to determine an optimum $N$ for all cases. But now, the designer has powerful tools. Studying a couple of examples, assuming different dual-rate controllers, some issues will be able to be extrapolated. 


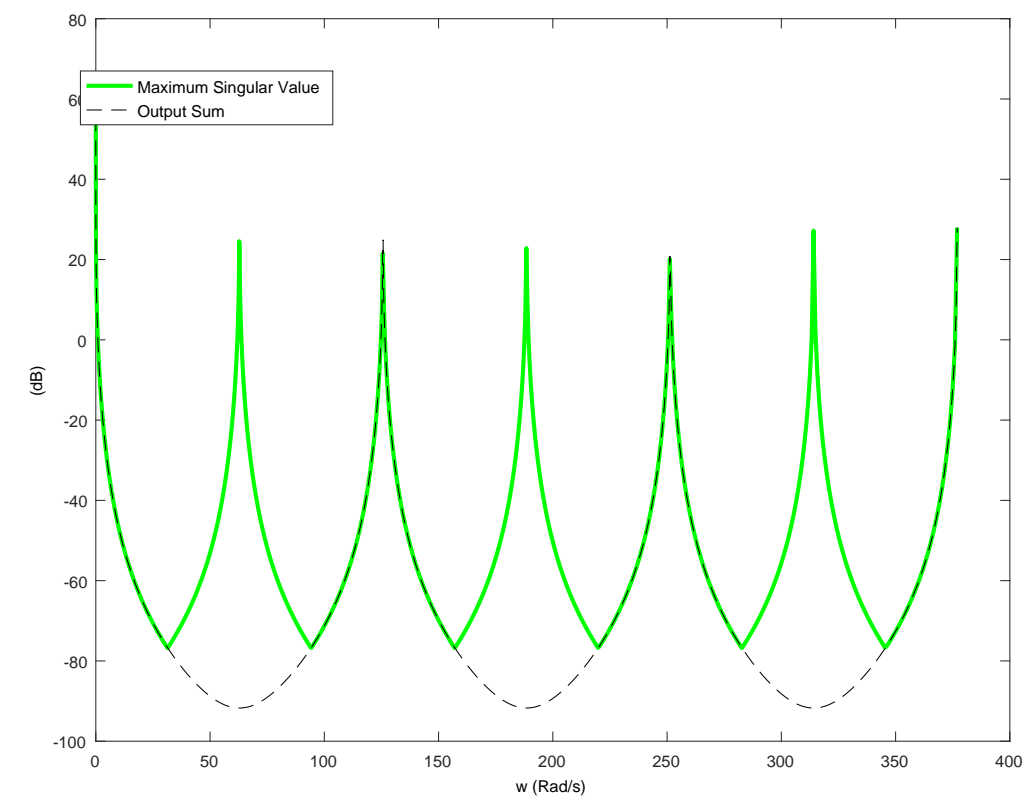

Figure 13. Metaperiod $T_{0}$ DRS Bode and the greatest Singular Value

\subsection{Example 1}

The following system is considered

$$
G(s)=\frac{1.5}{(s+0.5)(s+1.5)}
$$

controlled by a parallel structure PID

$$
G_{r}(s)=K_{p}\left(1+T_{d} s+\frac{1 / T_{i}}{s}\right)
$$

with $K_{p}=8, T_{d}=0.2$ and $T_{i}=8$ for desired specifications. Due to problems in measurement frequency, the sampling time at process output is restricted to $T_{0}=$ $0.3 \mathrm{sec}$. If the control scheme (case $N=1$ ) is analysed it leads to an unacceptable behaviour with excessive underdamping and slow response. The proposal is to apply the scheme in figure 14. The non-conventional controller with slow input $T_{0}$ and fast output $T_{0} / N$ is composed by a digital hold, $H^{T_{0}, T_{0} / N}$, which repeats every $T_{0}$ input $N$ times, and the $T_{0} / N$ discretization $G_{R}^{T_{0} / N}$ of the analog controller $G_{r}(s)$ using a known discretization technique ${ }^{4}$. With these conditions, $e\left(k T_{0}\right)=e\left(k T_{0}+T_{0} / N\right)=$ $\ldots=e\left(k T_{0}+(N-1) T_{0} / N\right)$ and using the procedure exposed in section 2 the lifting matrix can be obtained.

Therefore, it is possible to obtain both the root locus and the Bode diagram ${ }^{5}$ regarding the $T_{0}$ open loop system for different values of $N=1,2,3,4, \ldots$. Figures 15 , 16 and 17 depict the time response, the root locus and the Bode diagram respectively.

\footnotetext{
${ }^{4}$ In this case the formula exposed in (Iserman 1990)

${ }^{5} \mathrm{It}$ is also possible to obtain the components Bode diagram if $G(s)$ fast intersampling is required. These diagrams must be done if ripple occurence is suspected
} 


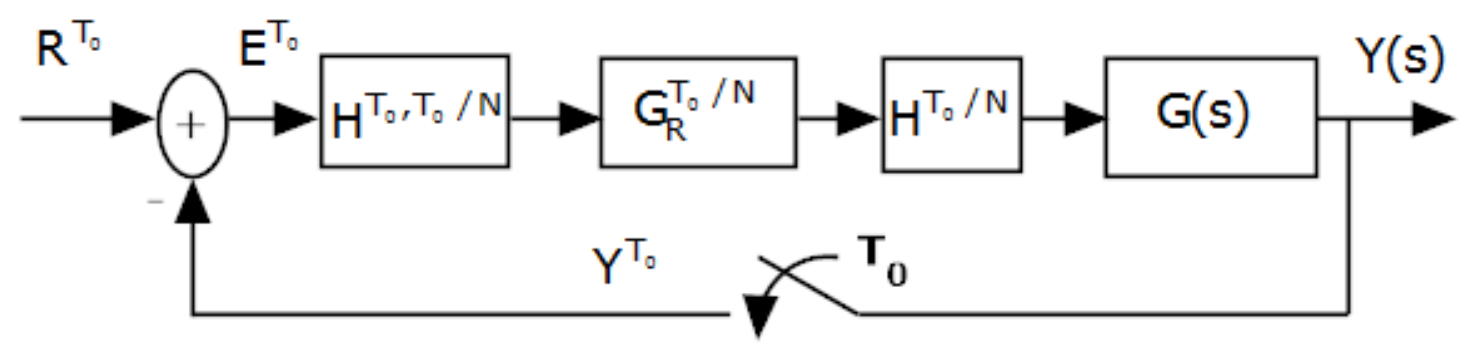

Figure 14. DR control scheme applied in example 1

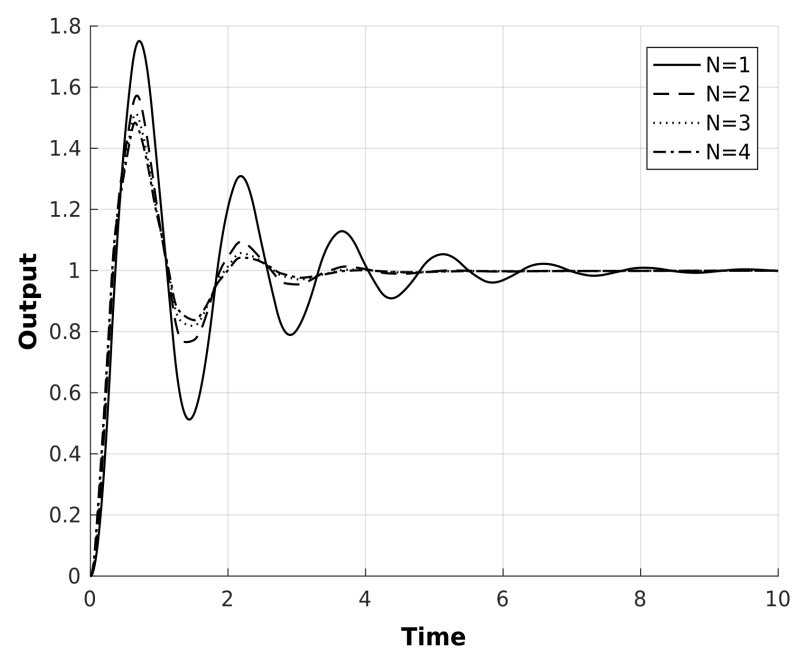

Figure 15. Time Response for different values of $N$

From these figures it is easy to understand that increasing the value of $N$ a better performance is obtained, although the most intensive improvement is from $N=1$ to $N=2$. The root locus is not able to clearly express the dynamic response because different closed loop poles and zeros prevent from an easy interpretation. The open loop Bode diagram is much clearer and it is observed how the margin phase is increasing with $N$ and therefore the underdamping is reduced. The gain margin is improved as well between $N=1$ and $N=2$, but among $N=2$ and successive values $N=$ $3,4, \ldots$ the magnitude margin decreases. As it has been said, this is not a recipe for all processes and specifications but these comments are usually valid for a large variety of common processes. By obtaining the closed loop model and using the aforementioned tool, the frequency response of the closed loop $Y / R$ was obtained as well (see figure 18). As it can be seen, the underdamping of the case $N=1$ is explained and it is deduced that the dual-rate cases present less high frequency noise rejection capability.

\subsection{Example 2}

Some works from author et al, (Salt and Albertos 2000, 2005), introduced a special structure for a Dual-Rate Control scheme in a Multi rate input problem (that is more frequent input than output of the process). The detailed explanation can be read at (Salt and Albertos 2005; Salt et al. 2014). In (Salt and Albertos 2005) the time- 


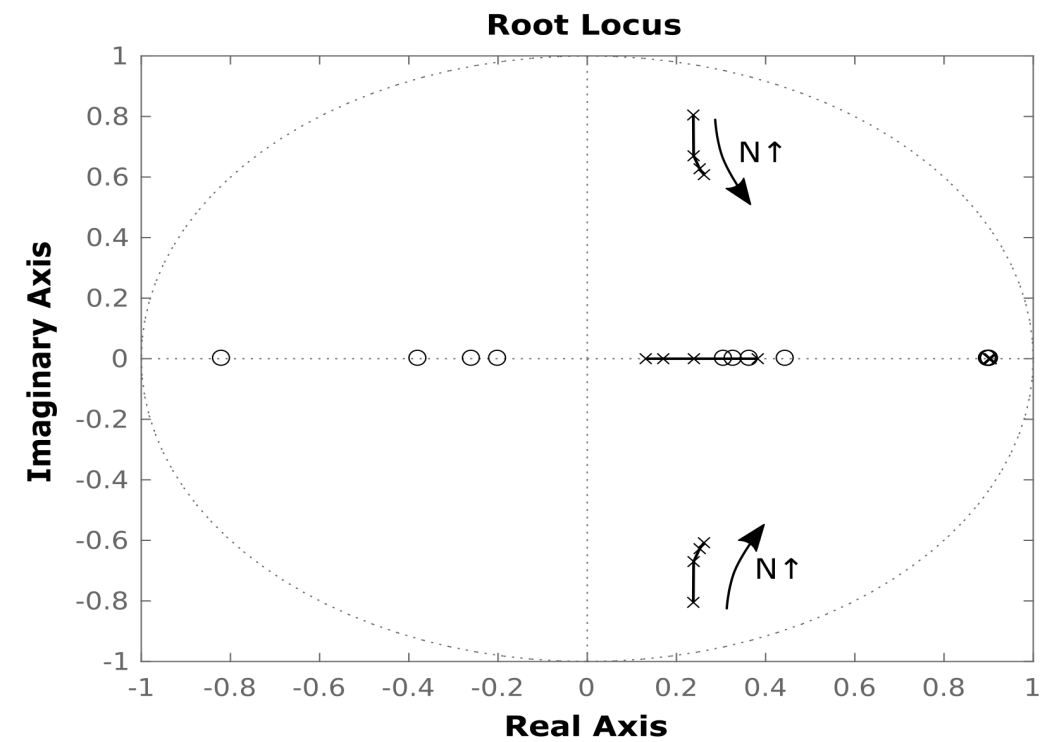

Figure 16. Root Locus for different values of $N$
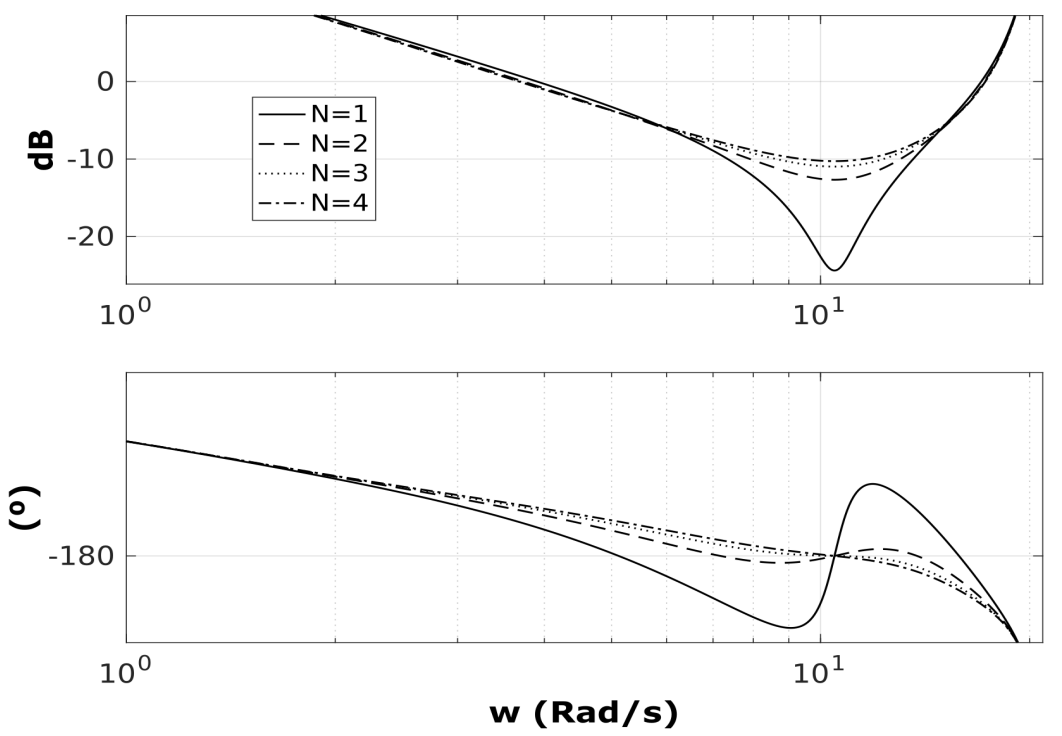

Figure 17. Open Loop Bode diagrams for different values of $N$ 

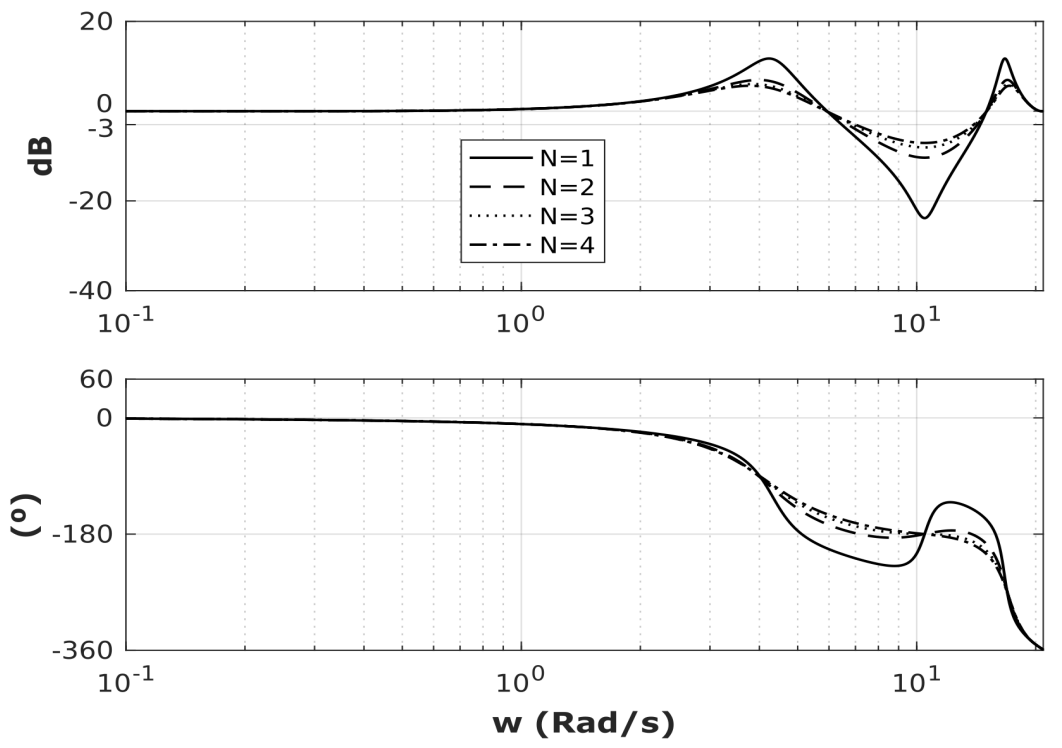

Figure 18. Closed Loop Bode diagrams for different values of $N$

design based procedure is exposed. Basically, this non-conventional structure dual-rate controller is composed by a slow part (working at the low frequency or measurement frequency) $G_{1}^{T_{0}}$, an expansion and digital holder $H^{T_{0}, T_{0} / N}$ to transform the slow signal output from slow dual-rate controller part, and a fast part (performing at updating control frequency or high frequency) $G_{2}^{T_{0} / N}$. In that contribution a model based design was introduced. The objective was to achieve a behaviour settled by continuous closed loop transfer function $M(s)$. In addition the ideal closed loop response follows the $M(s) \mathrm{ZOH}$ discretization at fast and slow frequencies. Some problems with ripple could occur but there are ways to avoid it as is pointed out in the cited contribution. The non conventional dual-rate controller parts are

$$
\begin{gathered}
G_{1}^{T_{0}}\left(z^{N}\right)=\frac{1}{1-M^{T_{0}}\left(z^{N}\right)} \\
G_{2}^{T_{0} / N}(z)=M^{T_{0} / N}(z) / G_{p}^{T_{0} / N}(z)=\frac{G_{R}^{T_{0} / N}(z)}{1+G_{R}^{T_{0} / N}(z) G_{p}^{T_{0} / N}(z)} \\
H^{T_{0}, T_{0} / N}(z)=\frac{R^{T_{0} / N}(z)}{\left(R^{T_{0}}\right)^{T}(z)}=\frac{1-z^{-N}}{1-z^{-1}}
\end{gathered}
$$

In figure 19 the configuration is depicted. Note that the algebra introduced in section 2 was considered.

There are different ways to design this DR controller. From the easiest one, that consists in assuming $G_{1}^{T_{0}}=1$ (like in example 1) and preserves the fast controller $G_{R}^{T_{0} / N}(z)$ for $G_{2}^{T_{0} / N}$, with unpredictable behaviour to more elaborated design procedures (see for instance (Salt et al. 2014)). In this contribution, the MR model-based 


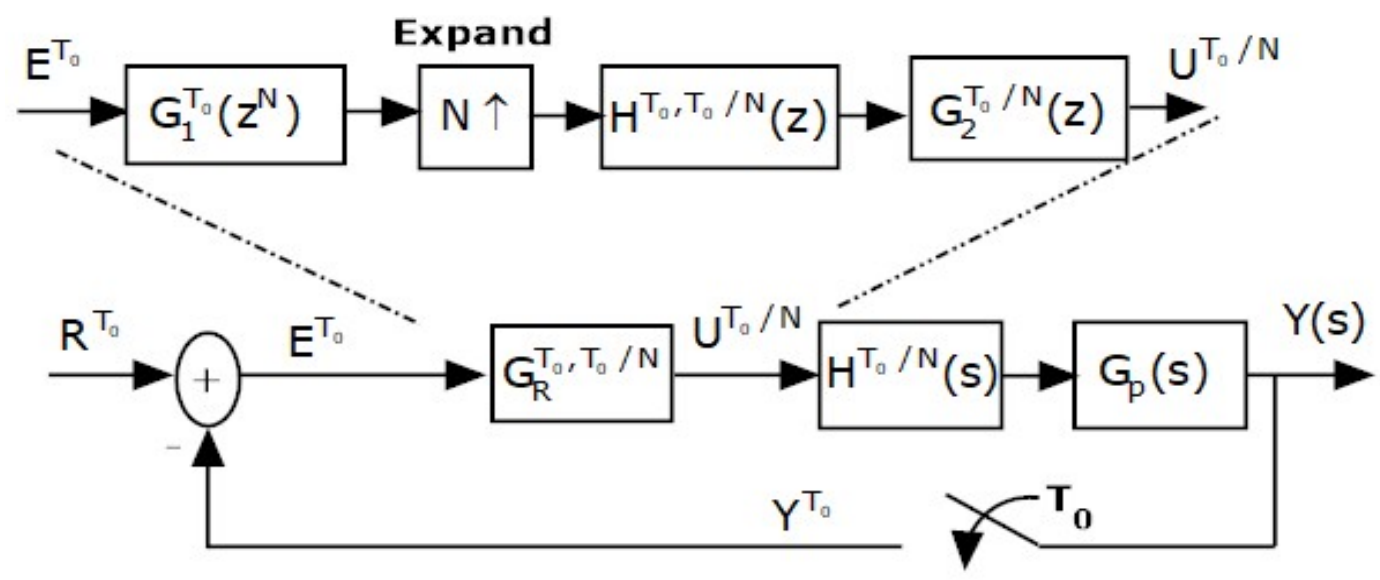

Figure 19. Closed Loop Dual-Rate Control. Non-conventional Dual-Rate Controller Structure used in example 2

controller will be assumed (Salt and Albertos 2005) (Figure 19).

Now, a specific example in this case is introduced. With the same plant and PID as presented in example 1 , the continuous closed loop transfer function is reached

$$
M(s)=\frac{2.4(s+4.66)(s+0.335)}{(s+0.33)\left(s^{2}+4 s+11.4\right)}
$$

With the same frame period, that is $T_{0}=0.3$ and successive values of $N=2,3,4$ the non-conventional controllers parts are computed. Specifically, the results for $N=2$ are

$$
\begin{aligned}
G_{1}^{T_{0}}\left(z^{2}\right) & =\frac{z^{3}-1.656 z^{2}+0.0 .9741 z-0.2671}{z^{3}-2.328 z^{2}+1.72 z-0.3913} \\
G_{2}^{T_{0} / N}(z) & =\frac{23.55 z^{4}-74.51 z^{3}+86.81 z^{2}-43.92 z+8.08}{z^{4}-1.402 z^{3}-0.2546 z^{2}+1.141 z-0.4677}
\end{aligned}
$$

The application of single and dual-rate controllers for different $N$ values leads to the results shown in figures 20, 21, 22 and 23.

As it can be seen, some similar results are obtained, that is, there is an improvement in time response which is suspected from the Root Locus. The open loop Bode diagrams confirm this intuition adding that with this kind of controllers the best step is from $N=1$ to $N=2$. For successive values of $N$ a similar phase margin but lower gain margin is obtained. The sensitivity is worse when the value of $N$ is higher.

\section{Conclusions}

In this contribution the interpretation of a new, proper and efficient way to obtain the frequency response of a DRS was introduced. It was also tested that the other methods for computing the MRS frequency response: MRS MIMO consideration and transfer function obtaining lead to partial or unacceptable results respectively. Some examples are introduced with the intention to clarify the understanding and consequences of this new method. This tool allows to properly ask some practical questions about MRS. 


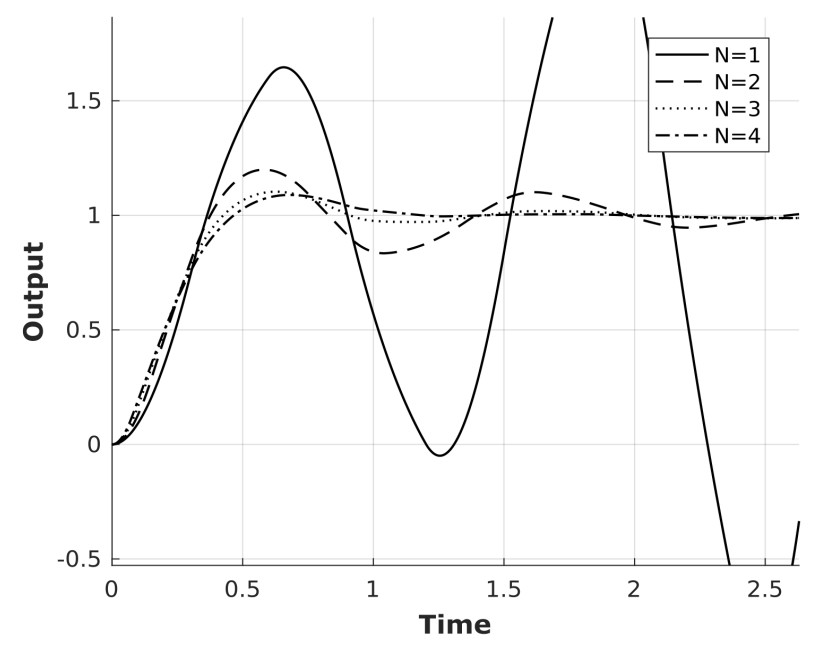

Figure 20. Time Response for different values of $N$

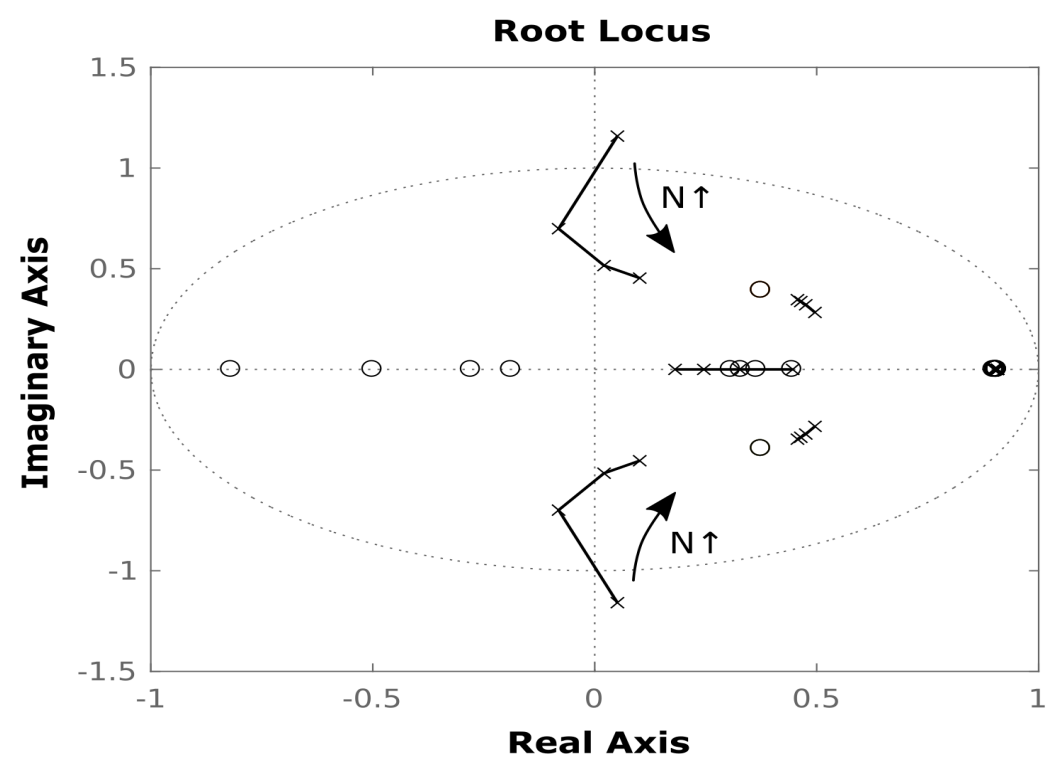

Figure 21. Root Locus for different values of $N$ 

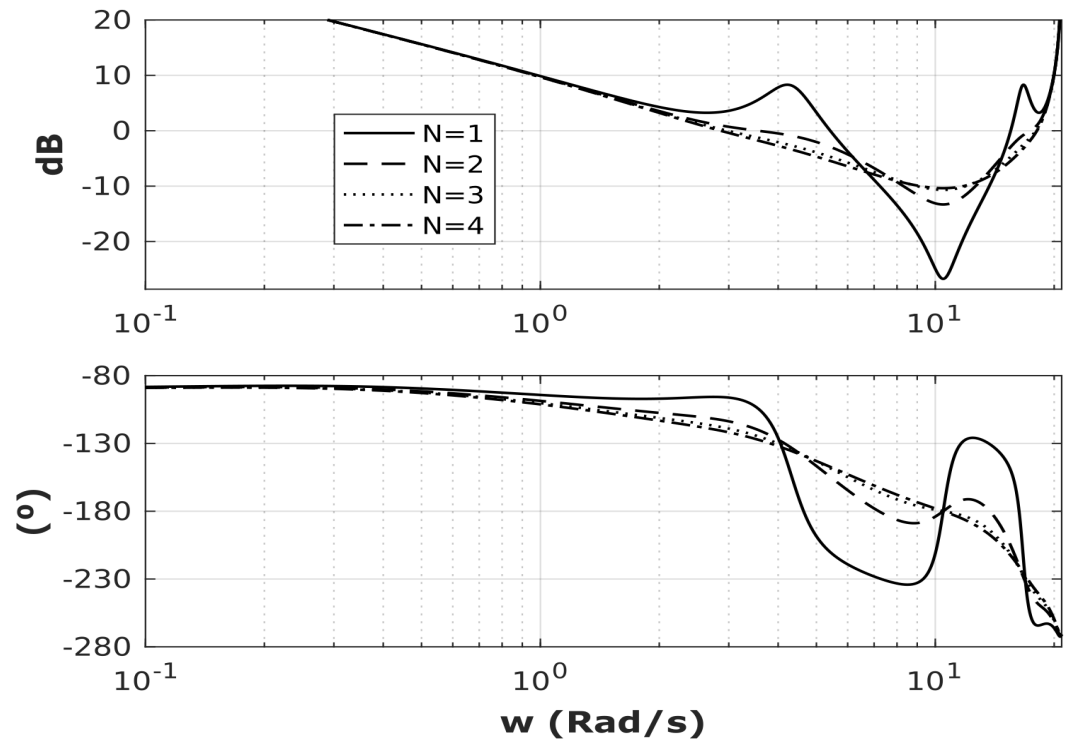

Figure 22. Open Loop Bode diagrams for different values of $N$

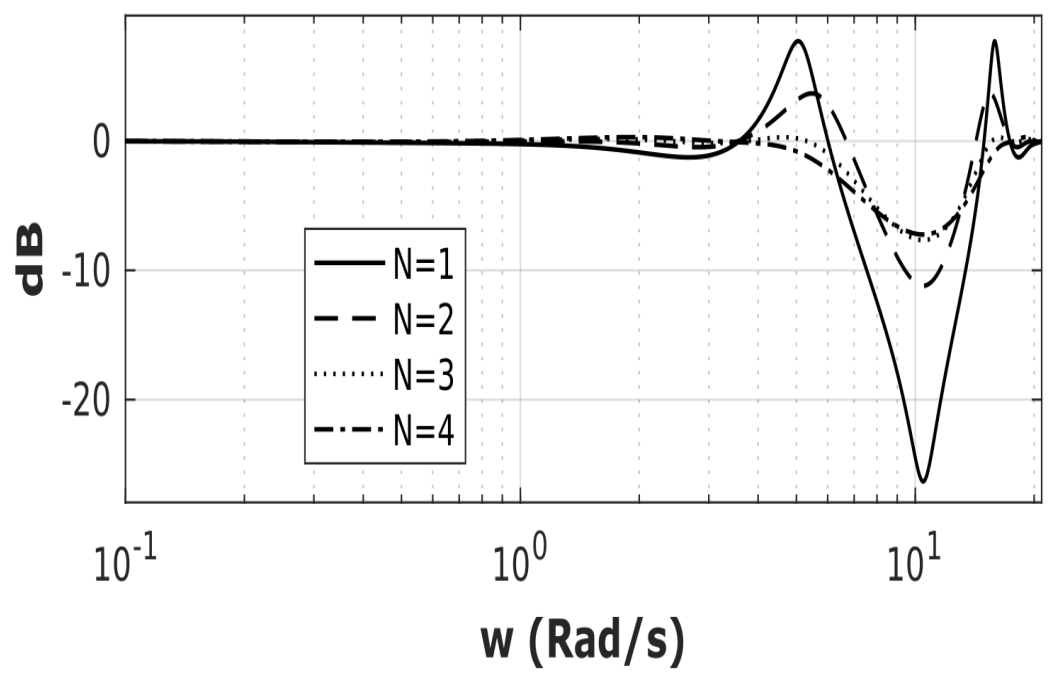

Figure 23. Closed Loop Bode diagrams for different values of $N$ 


\section{Disclosure statement}

No potential conflict of interest was reported by the authors.

\section{Notes on contributors}

Julián Salt received the M.Sc. degree in industrial engineering and the Ph.D. degree in control engineering from the Technical University of Valencia, Valencia, Spain, in 1986 and 1992, respectively. He is a Full Professor with the Technical University of Valencia. He has been teaching during 32 academic years (from 1986) diverse contents in grade master and doctoral courses all related to Simulation, Automation and Systems Control. He has been advisor of different grade and master thesis and $7 \mathrm{PhD}$ in the last 10 years. All of these works were related to Non-Conventional Sampling Systems. He has co-authored over 70 papers in journals and conferences. His current research interests include nonconventionally sampled control and networked control systems.

José Joaquín Alcaina received his M.Sc. degree in Automation and Industrial Computing from the Technical University of Valencia, Valencia, Spain, in 2015. He is currently completing his $\mathrm{PhD}$ studies in Automation, Robotics and Industrial Computer Science. His current research interests include networked control systems, packet-based dual-rate control and systems with low sensing ability.

\section{References}

Albertos, P. 1990. "Block multirate input-output model for sampled-data control systems." Automatic Control, IEEE Transactions on 35 (9): 1085-1088.

Bamieh, B., J.B. Pearson, B.A. Francis, and A. Tannenbaum. 1991. "A lifting technique for linear periodic systems with applications to sampled-data control." Systems $\& 3$ Control Letters 17 (2): 79-88.

Bu, Xianye, Hongli Dong, Fei Han, and Gongfa Li. 2018. "Event-triggered distributed filtering over sensor networks with deception attacks and partial measurements." International Journal of General Systems 47 (5): 522-534.

Chen, Feng Ding; Tongwen. June 2005. "Hierarchical identification of lifted state-space models for general dual-rate systems." Circuits and Systems I: Regular Papers, IEEE Transactions on [Circuits and Systems I: Fundamental Theory and Applications, IEEE Transactions on] 52 (6): 1179-1187.

Cimino, M., and P.R. Pagilla. 2010a. "Conditions for the ripple-free response of multirate systems using linear time-invariant controllers." Systems \& Control Letters 59 (8): 510-516.

Cimino, M., and P.R. Pagilla. 2010b. "Design of linear time-invariant controllers for multirate systems." Automatica 46 (8): 1315-1319.

Coffey, Thomas C, and Ivan J Williams. 1966. "Stability analysis of multiloop, multirate sampled systems." AIAA Journal 4 (12): 2178-2190.

Cuenca, Á., J. Salt, A. Sala, and R. Pizá. 2011. "A delay-dependent dual-rate PID controller over an ethernet network." Industrial Informatics, IEEE Transactions on 7 (1): 18-29.

Du, Y.Y., J.S.H. Tsai, H. Patil, L.S. Shieh, and Y. Chen. 2011. "Indirect identification of continuous-time delay systems from step responses." Applied Mathematical Modelling 35 (2): 594-611.

Friedland, B. 1959. Sampled Data Control Systems Containing Periodically Varying Numbers. United States Air Force, Office of Scientific Research. 
Hou, Nan, Hongli Dong, Weidong Zhang, Yurong Liu, and Fuad E Alsaadi. 2018. "Eventtriggered state estimation for time-delayed complex networks with gain variations based on partial nodes." International Journal of General Systems 47 (5): 477-490.

Iserman, R. 1990. "Digital Control." .

Kalman, Rudolf Emil, and JE Bertram. 1959. "General synthesis procedure for computer control of single-loop and multiloop linear systems (An optimal sampling system)." Transactions of the American Institute of Electrical Engineers, Part II: Applications and Industry 77 (6): 602-609.

Kranc, G. 1957. "Input-output analysis of multirate feedback systems." Automatic Control, IRE Transactions on 3 (1): 21-28.

Liu, Dan, Yurong Liu, and Fuad E Alsaadi. 2016. "A new framework for output feedback controller design for a class of discrete-time stochastic nonlinear system with quantization and missing measurement." International Journal of General Systems 45 (5): 517-531.

Meyer, R., and C. Burrus. 1975. "A unified analysis of multirate and periodically time-varying digital filters." Circuits and Systems, IEEE Transactions on 22 (3): 162-168.

Petrella, Roberto, and Marco Tursini. 2008. "An embedded system for position and speed measurement adopting incremental encoders." IEEE Transactions on industry applications 44 (5): 1436-1444.

Salt, J., and P. Albertos. 2005. "Model-Based Multirate Controllers Design." Control Systems Technology, IEEE Transactions on 13 (6): 988-997.

Salt, J., J. Torneno, and P. Albertos. 1993. "Modelling of non-conventional sampled data systems." Control Applications, 1993., Second IEEE Conference on 631-635.

Salt, Julián, and Pedro Albertos. 2000. "Multirate controllers design by rate decomposition." In Decision and Control, 2000. Proceedings of the 39th IEEE Conference on, Vol. 5, 48954900. IEEE.

Salt, Julián, Ángel Cuenca, Francisco Palau, and Sebastián Dormido. 2014. "A multirate control strategy to the slow sensors problem: An interactive simulation tool for controller assisted design." Sensors 14 (3): 4086-4110.

Salt, Julian, and Antonio Sala. 2014. "A new algorithm for dual-rate systems frequency response computation in discrete control systems." Applied Mathematical Modelling 38 (23): 5692-5704.

Thompson, P. M. 1986. "Gain and phase margins of multi-rate sampled-data feedback systems." International Journal of control 44 (3): 833-846.

Tsai, JSH, CM Chen, and LS Shieh. 1993. "Modelling of multirate feedback systems using uniform-rate models." Applied mathematical modelling 17 (1): 2-14.

Zhao, Di, Zidong Wang, Derui Ding, Guoliang Wei, and Fuad E Alsaadi. 2018. "PID outputfeedback control under event-triggered protocol." International Journal of General Systems 47 (5): 432-445. 